\title{
RESEARCH
}

\section{Genetics of aldosterone-producing adenomas with pathogenic KCNJ5 variants}

\author{
Antonio M Lerario1,*, Kazutaka Nanba2,*, Amy R Blinder², Sachiko Suematsu³, Masao Omura4, Tetsuo Nishikawa3, \\ Thomas J Giordano $1,5,6$, William E Rainey ${ }^{1,2}$ and Tobias Else ${ }^{1}$
}

1Division of Metabolism, Endocrinology, and Diabetes, University of Michigan, Ann Arbor, Michigan, USA 2Department of Molecular and Integrative Physiology, University of Michigan, Ann Arbor, Michigan, USA

${ }^{3}$ Endocrinology and Diabetes Center, Yokohama Rosai Hospital, Yokohama, Japan

${ }^{4}$ Medical Checkup Clinic, Minatomirai Medical Square, Sowa-Group, Yokohama, Japan

${ }^{5}$ Department of Pathology, University of Michigan, Ann Arbor, Michigan, USA

${ }^{6}$ Comprehensive Cancer Center, University of Michigan, Ann Arbor, Michigan, USA

Correspondence should be addressed to T Else: telse@umich.edu

*(A M Lerario and K Nanba contributed equally to this work)

\begin{abstract}
Somatic variants in genes that regulate intracellular ion homeostasis have been identified in aldosterone-producing adenomas (APAs). Although the mechanisms leading to increased aldosterone production in APA cells have been well studied, the molecular events that cause cell proliferation and tumor formation are poorly understood. In the present study, we have performed whole-exome sequencing (WES) to characterize the landscape of somatic alterations in a homogeneous series of APA with pathogenic KCNJ5 variants. In the WES analysis on 11 APAs, 84 exonic somatic events were called by 3 different somatic callers. Besides the KCNJ5 gene, only two genes (MED13 and ZNF669) harbored somatic variants in more than one APA. Unlike adrenocortical carcinomas, no chromosomal instability was observed by the somatic copy-number alteration and loss of heterozygosity analyses. The estimated tumor purity ranged from 0.35 to 0.67 , suggesting a significant proportion of normal cell infiltration. Based on the results of PureCN analysis, the KCNJ5 variants appear to be clonal. In conclusion, in addition to KCNJ5 somatic pathogenic variants, no significant somatic event that would obviously explain proliferation or tumor growth was observed in our homogeneous cohort of KCNJ5-mutated APA. The molecular mechanisms causing APA growth and tumorigenesis remain to be elucidated.
\end{abstract}

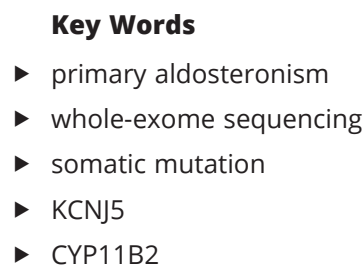

Endocrine-Related Cancer (2019) 26, 463-470

\section{Introduction}

Primary aldosteronism (PA) has been recognized as a major cause of endocrine and drug-resistant hypertension that is associated with significant morbidity and mortality (Funder et al. 2016). Aldosterone-producing adenoma (APA) is one of the major subtypes of PA (Young 2007). Over the past decade, there has been a considerable focus on defining the genetic bases of excess aldosterone production in PA. After the initial identification of somatic and germline KCNJ5 variants in PA patients (Choi et al. 2011), different germline and somatic variants have been identified in familial and sporadic forms of PA (Zennaro et al. 2017, Monticone et al. 2018). The immediate (c) 2019 Society for Endocrinology Published by Bioscientifica Ltd. Printed in Great Britain 
functional consequences of these alterations are disruption of intracellular ion homeostasis, which ultimately lead to increased aldosterone synthase (CYP11B2) transcription and inappropriate aldosterone production (Zennaro et al. 2017, Monticone et al. 2018). KCNJ5 mutations are the most common somatic variants in APA with greater frequency in East Asian populations (Zennaro et al. 2017). However, the mechanism by which these variants provide growth advantage to adrenocortical cells and promote APA formation remains poorly understood.

Recent studies have demonstrated that in patients with PA and multiple adrenal nodules, somatic variants in different aldosterone-driver genes can be present in distinct CYP11B2-expressing, but not in CYP11B2-negative nodules within the same adrenal (Dekkers et al. 2014, Fernandes-Rosa et al. 2015, Nanba et al. 2016). Additionally, in vitro studies indicate that mutated KCNJ5 channels do not confer growth advantage when expressed in the human adrenocortical HAC15 cell line (Oki et al. 2012). These observations suggest that the processes of nodule formation and autonomous aldosterone production may be independent events - the so-called 'two-hit' model (Zennaro et al. 2017). According to this model, an initial somatic event that confers growth advantage would induce tumor formation and a second mutational event (in any of the already characterized genes associated with APA) would be responsible for increased aldosterone production. However, no recurrent secondary mutations in growth-promoting genes have been reported in APA with mutations in aldosterone-driver genes so far. In this study, we used whole-exome sequencing (WES) to characterize the landscape of somatic alterations in a homogeneous series of APAs with KCNJ5 variants. We were particularly interested in identifying novel recurrent mutations and somatic alterations that may contribute to APA tumorigenesis and in further characterizing KCNJ5-mutated tumors in terms of tumor purity, ploidy and clonal composition.

\section{Patients and methods}

\section{Patients and samples}

Fourteen APA from 14 patients with PA who underwent adrenalectomy at the University of Michigan or Yokohama Rosai Hospital were included in the present study. To study genetic alterations related to APA tumorigenesis, relatively large APAs (median: $30 \mathrm{~mm}$, interquartile range: $23.8-30.0 \mathrm{~mm}$ ) were studied. Clinical characteristics of studied patients are shown in Supplementary Table 1 (see section on supplementary data given at the end of this article). For somatic variant identification, control germline DNA either from peripheral blood $(n=5)$ or matched adjacent normal adrenal $(n=6)$ was used based on the availability. Peripheral blood or matched adjacent normal adrenal and APA tissue DNA was isolated using commercial kits (Nucleospin Blood (MachereyNagel), Nucleospin Tissue (Macherey-Nagel) or AllPrep DNA/RNA/Protein kit (Qiagen)). This study was approved by Institutional Review Board of the University of Michigan and Yokohama Rosai Hospital. Either a written informed consent was obtained from patients before the use of their biospecimens for the analysis (24-51) or a waiver of informed consent was granted for the use of specimens at the University of Michigan (HUM00083056).

\section{Immunohistochemistry}

Formalin-fixed paraffin-embedded tissue blocks were available from all but one patient (Case 10, APA-Y182). Immunohistochemistry (IHC) was performed using specific antibodies against CYP11B2 (mouse monoclonal, Millipore, \#MABS1251) (Gomez-Sanchez et al. 2014), 11ß-hydroxylase (CYP11B1, rat monoclonal) (Gomez-Sanchez et al. 2017), 17 $\alpha$-hydroxylase (CYP17A1, mouse monoclonal) (Dharia et al. 2004), 33-hydroxysteroiddehydrogenase/ $\Delta 4$-5 isomerase type II (HSD3B2, mouse monoclonal) (GomezSanchez et al. 2017) and $\beta$-catenin (mouse monoclonal, BD Biosciences, \#610153) essentially as described previously (Monticone et al. 2012, Nanba et al. 2016).

\section{Whole-exome sequencing}

DNA was fragmented to $300 \mathrm{bp}$ using standard Covaris sonication (Covaris). Fragmented DNA was then prepared as a standard Illumina library using IntegenX (now Wafergen) reagents on the Apollo instrument, where the fragments were end repaired, A tailed and adapter ligated. Then, the samples were PCR amplified. Libraries were checked for quality and quantity by TapeStation (Agilent) and pooled for capture - six samples per pool. Each pool underwent exome capture using the Roche Nimblegen SeqCap EZ v3.0 using manufacturer's protocols (Roche NimbleGen). In brief, a total of $1 \mu \mathrm{g}$ prepared libraries was hybridized to the SeqCap EZ library for $72 \mathrm{~h}$ and then washed, and the captured DNA was recovered using Streptavidin Dynabeads (Life Technologies). The captured DNA was then amplified by PCR. The purified captured DNA was then clustered using the cBot (Illumina) and sequenced on a HiSeq 2500 (Illumina) high-output 
paired-end $125 \mathrm{bp}$ run using version 4 reagents and manufacturer's protocols.

\section{Data analysis}

Paired-end reads were aligned to the hg19 assembly of the human genome using bwa-mem (Li 2013). Aligned reads were sorted and duplicates were marked with bamsort and bammarkduplicate tools, respectively, from biobambam2 (Tischler \& Leonard 2014). Indel realignment and quality score recalibration were performed with GATK (McKenna et al. 2010). Coverage metrics and quality-control analysis of the alignments were performed with Qualimap2 (Okonechnikov et al. 2016). As an additional qualitycontrol measure, we used NGSCheckMate (Lee et al. 2017) to rule out cross-contamination, sample mislabeling and unexpected similarities among samples from different patients. Somatic variants were called with three different callers: MuTect2, Lancet and LoFreq (Wilm et al. 2012, Cibulskis et al. 2013, Narzisi et al. 2017). Somatic calls were annotated with Annovar (Wang et al. 2010). We considered somatic variants all those called by at least two of the callers. We used the Integrative Genomics Viewer (IGV) to visually confirm the somatic alterations and the reported allele frequencies. We compared the final list of somatic variants to an internal database generated from other projects using our in-house pipeline to further reduce the possibility of calling recurrent sequencing artifacts. For samples which paired germline DNA were not available, we calculated the allele frequencies of pathogenic KCNJ5 variants from the VCF files generated with samtools mpileup/bcftools (Li et al. 2009, Danecek et al. 2011). Finally, we used PureCN (Riester et al. 2016) to perform somatic copy-number alteration (SCNA) and loss of heterozygosity (LOH) analyses and to estimate tumor purity, ploidy and clonal composition. PureCN infers tumor purity and ploidy by simultaneously analyzing depth-of-coverage and variant allele frequencies from germline and tumor DNA.

\section{Results}

\section{Histologic characteristics of KCNJ5-mutated APA}

In accordance with previous studies (Azizan et al. 2012, Monticone et al. 2015), the KCNJ5-mutated tumor cells were mainly composed of lipid-rich clear cells. IHC was performed to assess the tumor expression of CYP11B2, which is essential for aldosterone biosynthesis, and to confirm the tumor cell capacity to produce aldosterone (Fig. 1A and C). IHC for other steroidogenic enzymes, including CYP17A1, CYP11B1 and HSD3B2, was also performed $(n=13)$. The CYP11B2 expression intensity was variable and most commonly exhibited a heterogeneous expression pattern (Supplementary Table 2). APA tumor cells also showed strong expression of HSD3B2 (Fig. 1D). The expression of CYP17A1 and CYP11B1, which are required for cortisol synthesis, was also observed in KCNJ5-mutated APA with some degree of variability (Fig. 1E and F, Supplementary Table 2). The results of $1 \mathrm{mg}$ dexamethasone suppression test were available in ten cases (Supplementary Table 1). Of those, three cases showed non-suppressed cortisol after a dexamethasone suppression test (serum cortisol levels higher than $1.8 \mu \mathrm{g} / \mathrm{dL}$ ). The APAs from those patients exhibited positive but variable expression of CYP17A1 and CYP11B1. We further performed IHC for $\beta$-catenin to determine a potential contribution to tumorigenesis or growth. The $\beta$-catenin IHC was not successful in one case (Case 1$)$. All other cases $(n=12)$ showed similar patterns of predominantly membranous staining, indicating the absence of $\mathrm{Wnt} / \beta$-catenin activation in the majority of the tumor cells (Supplementary Fig. 1). Only a few cases showed focal nuclear and/or cytoplasmic reactivity, which did not reach the staining intensity, usually observed in adrenocortical carcinomas with genetic changes known to activate the $\mathrm{Wnt} / \beta$-catenin pathway.

\section{Somatic mutation analysis}

We performed WES on 11 APA with paired germline DNA and 3 unpaired APA samples. The mean coverage and the percentage of targeted regions that were covered at least $20 \times$ were $90.45 \times$ (range 67.47-118.95×) and $91.12 \%$ (range 88.59-92.81\%) for tumors and 89.45× (range 63.46-120.11×) and 91.45\% (range 89.07-92.65\%) for germline. Our NGSCheckMate analysis ruled out crosscontamination, sample mislabeling and unexpected similarities among samples from different patients (Supplementary Fig. 2). We identified 120 exonic somatic events that were called at least by 2 callers, including 109 single-nucleotide variants (SNVs) (22 synonymous, 81 nonsynonymous and 6 stopgain), 6 indels (4 frameshift deletions, 1 frameshift insertion and 1 nonframeshift insertion) and 5 splicing site variants, averaging 10.9 events per sample (range 5-16). Among these, 84 events were called by the three callers, 32 were called by Mutect 2 and Lofreq, 3 by Mutect 2 and Lancet and 1 by Lancet and LoFreq (Supplementary Fig. 3). Apart from KCNJ5 variants, which were detected by the three 


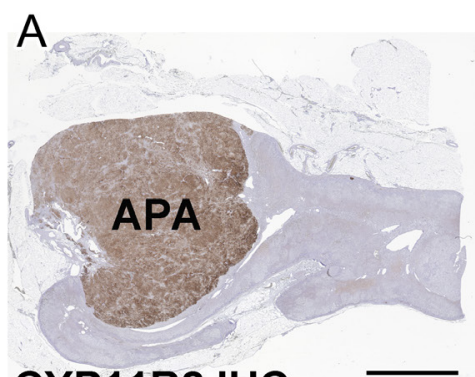

CYP11B2 IHC

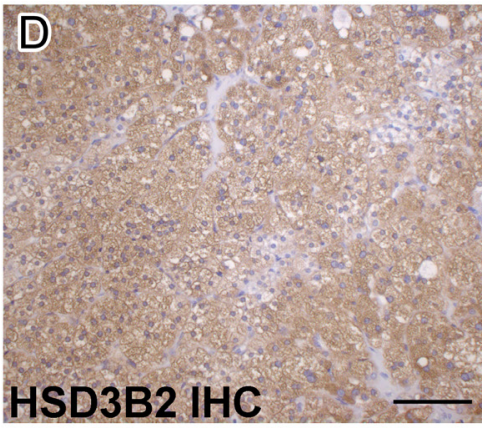

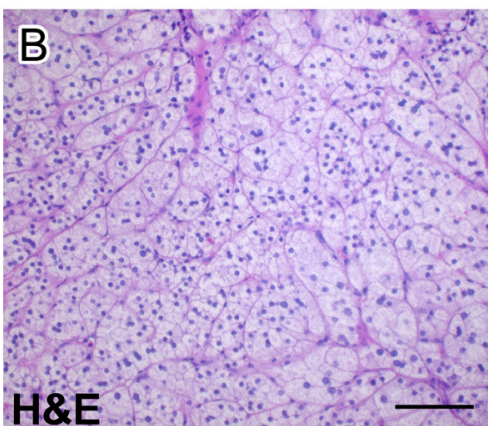
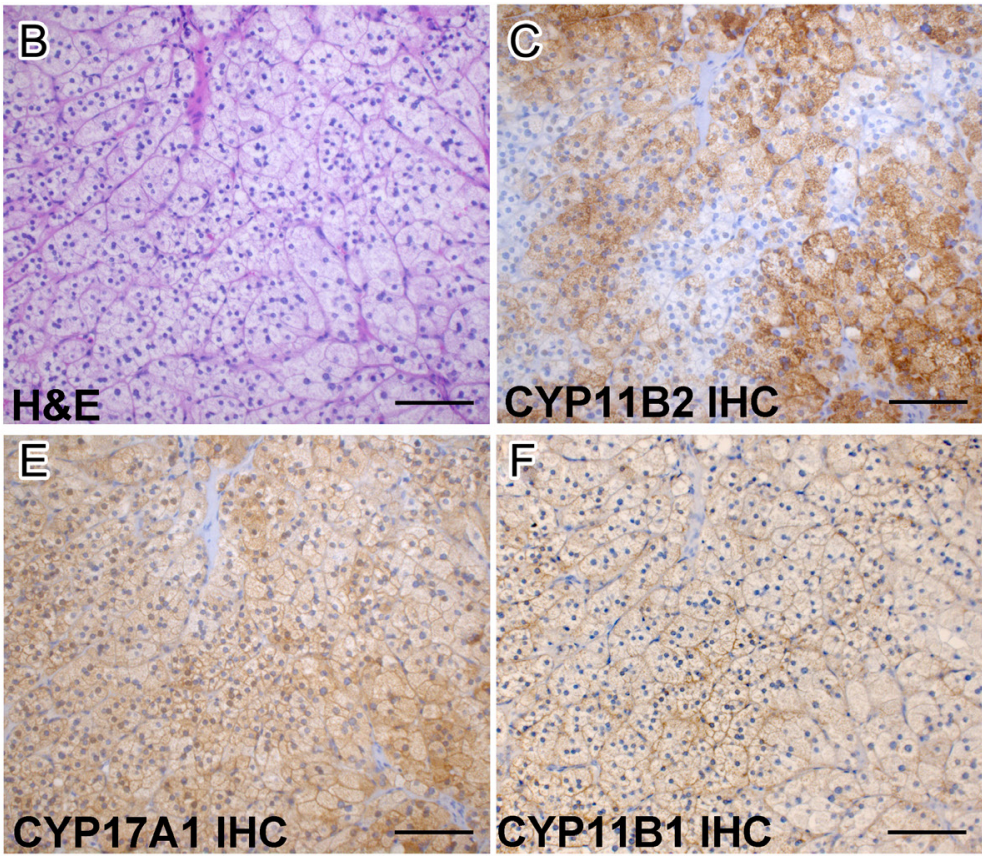

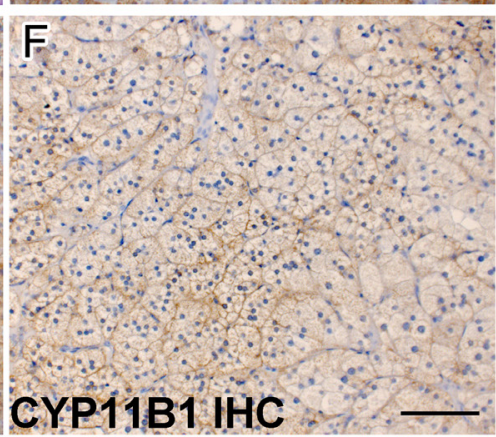

\section{Figure 1}

Representative histologic characteristics of KCNJ5-mutated aldosterone-producing adenoma. (A) Low magnification view of the adrenal gland with CYP11B2 IHC (Case 5, APA-Y80). The APA was identified by CYP11B2 IHC. Scale bar, $5 \mathrm{~mm}$. (B, C, D, E and F) High magnification view of the APA, (B) H\&E, (C) CYP11B2 IHC, (D) HSD3B2 IHC, (E) CYP17A1 IHC, (F) CYP11B1 IHC. Scale bars, $100 \mu \mathrm{m}$. APA, aldosterone-producing adenoma; IHC,

immunohistochemistry; H\&E, hematoxylin and eosin staining.

callers in all samples, only two other genes had variants in more than one APA: MED13 (NM_005121:exon26:c. T5866G:p.Ser1956Ala, (APA-78 and APA-86)) and ZNF669 (NM_001142572:exon4:c.A1052C:p.Glu351Ala, (APA-78 and APA-86)) (Supplementary Figs 4, 5, 6 and 7). Of note, the MED13 and ZNF669 variants were not confirmed by direct Sanger sequencing likely due to the low variant frequencies.

Among the identified mutated genes, six of them have been reported as cancer-associated (Futreal et al. 2004) (COSMIC v85): JAK1, NAB2, FOXP1, ATM, MEN1 and USP6. Mutations in these genes have been shown to affect cellular processes that are frequently dysregulated in cancer and are thought to be tumor promoting. Additionally, we identified variants in PDE11A and CYP17A1, genes that are essential components of the adrenal steroidogenesis machinery and have been implicated in other adrenal diseases, such as forms of adrenal micronodular hyperplasia, cortisol-producing adenomas (CPAs) and congenital adrenal hyperplasia. Finally, we identified variants in other ionic channels such as CACNA1E and KCNA1 (Supplementary Tables 3, 4,5 and 6). Noteworthy, exome sequencing confirmed the presence of four previously identified KCNJ5 variants in out cohort: c.T503G (p.L168R), c.G451A (p.G151R), c.G451C
(p.G151R) and c.477_478insATT (p.T149delinsTI) with allele frequencies ranging from 14 to $48 \%$ (Table 1).

\section{SCNA and LOH analyses}

In agreement with previous study (Choi et al. 2011), our SCNA and LOH analyses revealed that unlike a subset of ACA with CTNNB1 mutations and adrenocortical carcinomas (ACCs) (Goh et al. 2014, Zheng et al. 2016), KCNJ5-mutated APA did not exhibit chromosomal instability (characterized by arm-level segmental loss and gains and/or alterations in ploidy). The diploid heterozygous state was maintained in all samples (Table 1 and Supplementary Fig. 8).

\section{Tumor purity and clonal composition}

KCNJ5-mutated APAs appear to bear a significant proportion of normal cell infiltration. Tumor purity in our cohort ranged from 0.35 to 0.67 , which contrasts with ACC that exhibit high purity according to a recent molecular profiling study (Zheng et al. 2016). Furthermore, PureCN analysis also demonstrated that KCNJ5 variants are heterozygous and likely clonal in all tumors, suggesting that these variants are early events in adenoma formation (Table 1 and Supplementary Fig. 8). 
Table 1 Genetic characteristics of KCNJ5-mutated aldosterone-producing adenoma.

\begin{tabular}{l}
\hline Case \\
\hline 1 \\
2 \\
3 \\
4 \\
5 \\
6 \\
7 \\
8 \\
9 \\
10 \\
11 \\
12 \\
13 \\
14
\end{tabular}

\begin{tabular}{ccc}
$\begin{array}{c}\text { Tumor } \\
\text { purity }\end{array}$ & & $\begin{array}{c}\text { Tumor } \\
\text { ploidy }\end{array}$ \\
\cline { 1 - 1 } 0.57 & & 2.00 \\
0.64 & & 2.00 \\
0.67 & & 2.00 \\
0.64 & & 2.00 \\
0.36 & & 2.00 \\
0.59 & & 1.98 \\
NA & & NA \\
0.55 & & 2.00 \\
NA & & NA \\
0.35 & & 1.99 \\
0.55 & & 2.02 \\
NA & NA \\
0.59 & 2.00 \\
0.58 & 2.00 \\
\hline
\end{tabular}

\begin{tabular}{l}
\hline KCNJ5 mutation \\
\hline p.G151R \\
p.L168R \\
p.L168R \\
p.T149delinsTI \\
p.L168R \\
p.G151R \\
p.G151R \\
p.G151R \\
p.L168R \\
p.L168R \\
p.L168R \\
p.G151R \\
p.L168R \\
p.G151R
\end{tabular}

\begin{tabular}{c}
\hline $\begin{array}{c}\text { KCNJ5 mutation } \\
\text { allele frequency }\end{array}$ \\
\hline 0.44 \\
0.29 \\
0.37 \\
0.34 \\
0.14 \\
0.26 \\
0.24 \\
0.31 \\
0.48 \\
0.26 \\
0.18 \\
0.22 \\
0.35 \\
0.40 \\
\hline
\end{tabular}

\begin{tabular}{c}
\hline $\begin{array}{c}\text { \# of mutated } \\
\text { alleles per cell }\end{array}$ \\
\hline 1 \\
1 \\
1 \\
1 \\
1 \\
1 \\
$\mathrm{NA}$ \\
1 \\
$\mathrm{NA}$ \\
1 \\
1 \\
$\mathrm{NA}$ \\
1 \\
1
\end{tabular}

\begin{tabular}{l}
\hline Clonality \\
\hline Clonal \\
Clonal \\
Clonal \\
Clonal \\
Clonal \\
Clonal \\
NA \\
Clonal \\
NA \\
Clonal \\
Clonal \\
NA \\
Clonal \\
Clonal
\end{tabular}

aDNA from matched adjacent normal was used as a control. bPeripheral blood DNA was used as a control. NA, not available.

\section{Discussion}

In recent years, high-throughput molecular profiling studies have identified recurrent variants targeting different regulators of intracellular calcium homeostasis, including KCNJ5, ATP1A1, ATP2B3 and CACNA1D in over half of APA. Despite the well-characterized effects of these variants in abnormal CYP11B2 expression and aldosterone production, the contribution to cell growth remain poorly understood (Zennaro et al. 2017). So far, a growth-promoting effect of KCNJ5 variants has not been demonstrated in vitro studies using HAC15 cells (Oki et al. 2012). Although it is difficult to assess a cell proliferation effect of these variants using highly proliferative H295R/HAC15 cells, which have a $\beta$-catenin mutation (Tissier et al. 2005), these in vitro studies suggest that alternative mechanisms might play a role in tumor formation. Interestingly, areas of cortical cell hyperplasia and other nodular lesions are frequently observed near APA. These areas are usually CYP11B2 negative and do not bear aldosterone-driving gene variants (Dekkers et al. 2014). Furthermore, APAs often exhibit a heterogeneous immunostaining for CYP11B2 (Nakamura et al. 2014), suggesting that mutated cells could be subclonal. Based on these observations, a 'two-hit' model in which tumor growth and increased aldosterone production are caused by independent molecular hits has been proposed (Zennaro et al. 2017). In fact, we observed at least two variants of uncertain significance in genes that have been previously associated with adrenocortical tumorigenesis: the MEN1 NM_130804:exon3:c.T364C (p.Ser122Pro) and the PDE11A NM_001077197:exon5:c.C514T (p.Arg172Cys) variants, both predicted to be pathogenic by SIFT, polyphen 2 and CADD. However, both of these variants were somatic and, according to our PureCN analysis, none of these variants are in focal areas of $\mathrm{LOH}$, suggesting that both are 'passenger' events. Although somatic variants in MED13 and ZNF669 were identified in more than one APA, the pathological role of these variants is unclear. MED13 is a component of the Mediator Complex, a multiprotein complex critical for transcriptional activation. Mutations and altered expression of different Mediator components have been described in rare neurodevelopmental disorders and in a broad spectrum of benign and malignant tumors (Clark et al. 2015). The function and physiological relevance of ZNF669 are currently unknown. Noteworthily, we did not identify variants in other genes previously described in APA, such as CTNNB1 (encoding $\beta$-catenin), suggesting a dominant role for KCNJ5 variants. These results are consistent with previous studies on APA genetics, in which KCNJ5 and CTNNB1 variants were mutually exclusive (Akerstrom et al. 2016, Wu et al. 2017). Previous reports have demonstrated that the activation of $\mathrm{Wnt} / \beta$-catenin pathway is frequent in APA regardless of the mutation status of CTNNB1 (Boulkroun et al. 2011, Berthon et al. 2014). However, the nonactived state of $\beta$-catenin was predominantly observed in our cohort, suggesting that, unlike ACC (Mete et al. 2018), activation of Wnt/ $\beta$-catenin pathway may not be a common feature of $K C N J 5$-mutated APA. For better understanding of the role of $\mathrm{Wnt} / \beta$-catenin signaling pathway activation in APA development, further analysis including APA with variants in other aldosteronedriver genes, such as CACNA1D, ATP1A1, and ATP2B3 will be needed. 
Finally, our clonality analysis suggests that KCNJ5mutated cells are a clonal population within the tumor rather than a subclone that acquired the ability to secrete aldosterone. Certain KCNJ5 variants, including p.G151R and p.T158A, appear to have a potential impact on cell proliferation considering the fact that these variants have been identified both in sporadic APAs (as somatic variants) and in patients with massive hyperplasia (as germline variants), whereas a KCNJ5 variant p.G151E has only been documented in individuals without massive adrenal hyperplasia as a germline variant (Choi et al. 2011, Mulatero et al. 2012, Scholl et al. 2012). Furthermore, in an early-onset PA patient, the identical somatic KCNJ5 p.G151R variant has been identified in multiple CYP11B2expressing hyperplastic lesions and APA in bilateral adrenals and the variant has not been observed in nonhyperplastic lesions (Tamura et al. 2017), supporting the potential role of the KCNJ5 variant on cell proliferation.

Taken together, our results do not support the proposed 'two-hit' model. However, since our study included only a homogeneous population of APA with KCNJ5 variants, the findings cannot be generalized. It is also important to note that the use of DNA from adrenal tissue adjacent to the tumors could have limited detection of somatic mutations considering a previous observation that small nodules are sometimes present in adrenal tissue adjacent to APA (Boulkroun et al. 2010). The development of APA might be complex and heterogeneous. A recent case report describes the co-occurrence of a germline APC variant and a somatic KCNJ5 variant in a young PA patient with multinodular adrenal hyperplasia (Vouillarmet et al. 2016). In the resected adrenal from the patient, the KCNJ5 variant was identified only in a CYP11B2-expressing nodule, whereas biallelic APC inactivation due to $\mathrm{LOH}$ was observed in both CYP11B2-positive and -negative nodules (Vouillarmet et al. 2016), indicating 'two-hit' model as a possible mechanism of APA development at least for some cases. Epigenetic event may also be involved in adrenal nodule formation in some APAs. A recent study of integrated transcriptome and methylome analysis has demonstrated global hypomethylation in APA and upregulated genes with CpG hypomethylation in APA, including five potentially tumorigenesis-related genes (Murakami et al. 2015).

Interestingly, while our analysis is consistent with a relatively high proportion of normal cells in the tumors, we do not observe a significant infiltration of fibroblasts or inflammatory cells. In fact, although CYP11B2 expression is heterogeneous in some tumors, the majority of the cells, including CYP11B2-negative cells, exhibit morphological features consistent with steroidogenic cells and express steroidogenic enzymes such as CYP17A1, HSD3B2 and CYP11B1 (Fig. 1). This observation suggests that the normal cell type that infiltrates KCNJ5-mutated APA are normal steroidogenic cells, consistent with a role for paracrine factors in mediating tumor growth.

In conclusion, our study did not identify recurrent somatic events that could explain proliferation or tumor formation in APA with KCNJ5 variants. The mechanism by which KCNJ5-mutated tumors form and grow remains elusive.

\section{Supplementary data}

This is linked to the online version of the paper at https://doi.org/10.1530/ ERC-18-0364.

\section{Declaration of interest}

The authors declare that there is no conflict of interest that could be perceived as prejudicing the impartiality of the research reported.

\section{Funding}

This work was supported by grants from NHLBI (1R01HL130106) and American Heart Association (14SDG17990000) to T E, NIDDK (DK106618) to W E R and American Heart Association (17SDG33660447) to K N. This research is supported (in part) by the National Institute of Health through the University of Michigan Cancer Center Support Grant (5 P30 CA46592).

\section{Acknowledgements}

The authors thank Dr Robert Lyons at the University of Michigan DNA sequencing core for whole-exome sequencing. They also thank Michelle Vinco at the University of Michigan for assistance in case identification and sample preparation and Kristina Fields at the University of Michigan for technical assistance.

\section{References}

Akerstrom T, Maharjan R, Sven Willenberg H, Cupisti K, Ip J, Moser A, Stalberg P, Robinson B, Alexander Iwen K, Dralle H, et al. 2016 Activating mutations in CTNNB1 in aldosterone producing adenomas. Scientific Reports 6 19546. (https://doi.org/10.1038/srep19546)

Azizan EA, Lam BY, Newhouse SJ, Zhou J, Kuc RE, Clarke J, Happerfield L, Marker A, Hoffman GJ \& Brown MJ 2012 Microarray, qPCR, and KCNJ5 sequencing of aldosterone-producing adenomas reveal differences in genotype and phenotype between zona glomerulosa- and zona fasciculata-like tumors. Journal of Clinical Endocrinology and Metabolism 97 E819-E829. (https://doi. org/10.1210/jc.2011-2965)

Berthon A, Drelon C, Ragazzon B, Boulkroun S, Tissier F, Amar L, Samson-Couterie B, Zennaro MC, Plouin PF, Skah S, et al. 2014 $\mathrm{WNT}$ /beta-catenin signalling is activated in aldosterone-producing adenomas and controls aldosterone production. Human Molecular Genetics 23 889-905. (https://doi.org/10.1093/hmg/ddt484) 
Boulkroun S, Samson-Couterie B, Dzib JF, Lefebvre H, Louiset E, Amar L, Plouin PF, Lalli E, Jeunemaitre X, Benecke A, et al. 2010 Adrenal cortex remodeling and functional zona glomerulosa hyperplasia in primary aldosteronism. Hypertension 56 885-892. (https://doi. org/10.1161/HYPERTENSIONAHA.110.158543)

Boulkroun S, Samson-Couterie B, Golib-Dzib JF, Amar L, Plouin PF, Sibony M, Lefebvre H, Louiset E, Jeunemaitre X, Meatchi T, et al. 2011 Aldosterone-producing adenoma formation in the adrenal cortex involves expression of stem/progenitor cell markers. Endocrinology 152 4753-4763. (https://doi.org/10.1210/en.20111205)

Choi M, Scholl UI, Yue P, Bjorklund P, Zhao B, Nelson-Williams C, Ji W, Cho Y, Patel A, Men CJ, et al. $2011 \mathrm{~K}+$ channel mutations in adrenal aldosterone-producing adenomas and hereditary hypertension. Science 331 768-772. (https://doi.org/10.1126/science.1198785)

Cibulskis K, Lawrence MS, Carter SL, Sivachenko A, Jaffe D, Sougnez C, Gabriel S, Meyerson M, Lander ES \& Getz G 2013 Sensitive detection of somatic point mutations in impure and heterogeneous cancer samples. Nature Biotechnology 31 213-219. (https://doi.org/10.1038/ nbt.2514)

Clark AD, Oldenbroek M \& Boyer TG 2015 Mediator kinase module and human tumorigenesis. Critical Reviews in Biochemistry and Molecular Biology 50 393-426. (https://doi.org/10.3109/10409238.2015.1064854)

Danecek P, Auton A, Abecasis G, Albers CA, Banks E, DePristo MA, Handsaker RE, Lunter G, Marth GT, Sherry ST, et al. 2011 The variant call format and VCFtools. Bioinformatics 27 2156-2158. (https://doi.org/10.1093/bioinformatics/btr330)

Dekkers T, ter Meer M, Lenders JW, Hermus AR, Schultze Kool L, Langenhuijsen JF, Nishimoto K, Ogishima T, Mukai K, Azizan EA, et al. 2014 Adrenal nodularity and somatic mutations in primary aldosteronism: one node is the culprit? Journal of Clinical Endocrinology and Metabolism 99 E1341-E1351. (https://doi. org/10.1210/jc.2013-4255)

Dharia S, Slane A, Jian M, Conner M, Conley AJ \& Parker CR Jr 2004 Colocalization of P450c17 and cytochrome B5 in androgensynthesizing tissues of the human. Biology of Reproduction 71 83-88. (https://doi.org/10.1095/biolreprod.103.026732)

Fernandes-Rosa FL, Giscos-Douriez I, Amar L, Gomez-Sanchez CE, Meatchi T, Boulkroun S \& Zennaro MC 2015 Different somatic mutations in multinodular adrenals with aldosterone-producing adenoma. Hypertension 66 1014-1022. (https://doi.org/10.1161/ HYPERTENSIONAHA.115.05993)

Funder JW, Carey RM, Mantero F, Murad MH, Reincke M, Shibata H, Stowasser M \& Young WF Jr 2016 The management of primary aldosteronism: case detection, diagnosis, and treatment: an Endocrine Society clinical practice guideline. Journal of Clinical Endocrinology and Metabolism 101 1889-1916. (https://doi. org/10.1210/jc.2015-4061)

Futreal PA, Coin L, Marshall M, Down T, Hubbard T, Wooster R, Rahman N \& Stratton MR 2004 A census of human cancer genes. Nature Reviews Cancer 4 177-183. (https://doi.org/10.1038/nrc1299)

Goh G, Scholl UI, Healy JM, Choi M, Prasad ML, Nelson-Williams C, Kunstman JW, Korah R, Suttorp AC, Dietrich D, et al. 2014 Recurrent activating mutation in PRKACA in cortisol-producing adrenal tumors. Nature Genetics 46 613-617. (https://doi.org/10.1038/ ng.2956)

Gomez-Sanchez CE, Qi X, Velarde-Miranda C, Plonczynski MW, Parker CR, Rainey W, Satoh F, Maekawa T, Nakamura Y, Sasano H, et al. 2014 Development of monoclonal antibodies against human CYP11B1 and CYP11B2. Molecular and Cellular Endocrinology 383 111-117. (https://doi.org/10.1016/j.mce.2013.11.022)

Gomez-Sanchez CE, Lewis M, Nanba K, Rainey WE, Kuppusamy M \& Gomez-Sanchez EP 2017 Development of monoclonal antibodies against the human 3beta-hydroxysteroid dehydrogenase/isomerase isozymes. Steroids 127 56-61. (https://doi.org/10.1016/j. steroids.2017.08.011)
Lee S, Lee S, Ouellette S, Park WY, Lee EA \& Park PJ 2017 NGSCheckMate: software for validating sample identity in nextgeneration sequencing studies within and across data types. Nucleic Acids Research 45 e103. (https://doi.org/10.1093/nar/gkx193)

Li H 2013 Aligning sequence reads, clone sequences and assembly contigs with BWA-MEM. arXiv:1303.3997v1. [q-bio.GN].

Li H, Handsaker B, Wysoker A, Fennell T, Ruan J, Homer N, Marth G, Abecasis G, Durbin R \& Genome Project Data Processing Subgroup 2009 The Sequence Alignment/Map format and SAMtools. Bioinformatics 25 2078-2079. (https://doi.org/10.1093/ bioinformatics/btp352)

McKenna A, Hanna M, Banks E, Sivachenko A, Cibulskis K, Kernytsky A, Garimella K, Altshuler D, Gabriel S, Daly M, et al. 2010 The Genome Analysis Toolkit: a MapReduce framework for analyzing nextgeneration DNA sequencing data. Genome Research 20 1297-1303. (https://doi.org/10.1101/gr.107524.110)

Mete O, Gucer H, Kefeli M \& Asa SL 2018 Diagnostic and prognostic biomarkers of adrenal cortical carcinoma. American Journal of Surgical Pathology 42 201-213. (https://doi.org/10.1097/ PAS.0000000000000943)

Monticone S, Hattangady NG, Nishimoto K, Mantero F, Rubin B, Cicala MV, Pezzani R, Auchus RJ, Ghayee HK, Shibata H, et al. 2012 Effect of KCNJ5 mutations on gene expression in aldosteroneproducing adenomas and adrenocortical cells. Journal of Clinical Endocrinology and Metabolism 97 E1567-E1572. (https://doi. org/10.1210/jc.2011-3132)

Monticone S, Castellano I, Versace K, Lucatello B, Veglio F, GomezSanchez CE, Williams TA \& Mulatero P 2015 Immunohistochemical, genetic and clinical characterization of sporadic aldosteroneproducing adenomas. Molecular and Cellular Endocrinology $\mathbf{4 1 1}$ 146-154. (https://doi.org/10.1016/j.mce.2015.04.022)

Monticone S, Buffolo F, Tetti M, Veglio F, Pasini B \& Mulatero P 2018 Genetics in endocrinology: the expanding genetic horizon of primary aldosteronism. European Journal of Endocrinology 178 R101-R111. (https://doi.org/10.1530/EJE-17-0946)

Mulatero P, Tauber P, Zennaro MC, Monticone S, Lang K, Beuschlein F, Fischer E, Tizzani D, Pallauf A, Viola A, et al. 2012 KCNJ5 mutations in European families with nonglucocorticoid remediable familial hyperaldosteronism. Hypertension 59 235-240. (https://doi. org/10.1161/HYPERTENSIONAHA.111.183996)

Murakami M, Yoshimoto T, Nakabayashi K, Tsuchiya K, Minami I, Bouchi R, Izumiyama H, Fujii Y, Abe K, Tayama C, et al. 2015 Integration of transcriptome and methylome analysis of aldosteroneproducing adenomas. European Journal of Endocrinology 173 185-195. (https://doi.org/10.1530/EJE-15-0148)

Nakamura Y, Maekawa T, Felizola SJ, Satoh F, Qi X, Velarde-Miranda C, Plonczynski MW, Ise K, Kikuchi K, Rainey WE, et al. 2014 Adrenal CYP11B1/2 expression in primary aldosteronism: immunohistochemical analysis using novel monoclonal antibodies. Molecular and Cellular Endocrinology 392 73-79. (https://doi. org/10.1016/j.mce.2014.05.002)

Nanba K, Chen AX, Omata K, Vinco M, Giordano TJ, Else T, Hammer GD, Tomlins SA \& Rainey WE 2016 Molecular heterogeneity in aldosterone-producing adenomas. Journal of Clinical Endocrinology and Metabolism 101 999-1007. (https://doi. org/10.1210/jc.2015-3239)

Narzisi G, Corvelo A, Arora K, Bergmann E, Shah M, Musunuri R, Emde A, Robine N, Vacic V \& Zodi MC 2017 Lancet: genome-wide somatic variant calling using localized colored DeBruijn graphs. bioRxiv.

Oki K, Plonczynski MW, Luis Lam M, Gomez-Sanchez EP \& GomezSanchez CE 2012 Potassium channel mutant KCNJ5 T158A expression in HAC-15 cells increases aldosterone synthesis. Endocrinology 153 1774-1782. (https://doi.org/10.1210/en.2011-1733)

Okonechnikov K, Conesa A \& Garcia-Alcalde F 2016 Qualimap 2: advanced multi-sample quality control for high-throughput 
sequencing data. Bioinformatics 32 292-294. (https://doi.org/10.1093/ bioinformatics/btv566)

Riester M, Singh AP, Brannon AR, Yu K, Campbell CD, Chiang DY \& Morrissey MP 2016 PureCN: copy number calling and SNV classification using targeted short read sequencing. Source Code for Biology and Medicine 11 13. (https://doi.org/10.1186/s13029-0160060-z)

Scholl UI, Nelson-Williams C, Yue P, Grekin R, Wyatt RJ, Dillon MJ, Couch R, Hammer LK, Harley FL, Farhi A, et al. 2012 Hypertension with or without adrenal hyperplasia due to different inherited mutations in the potassium channel KCNJ5. PNAS 109 2533-2538. (https://doi.org/10.1073/pnas.1121407109)

Tamura A, Nishimoto K, Seki T, Matsuzawa Y, Saito J, Omura M, GomezSanchez CE, Makita K, Matsui S, Moriya N, et al. 2017 Somatic KCNJ5 mutation occurring early in adrenal development may cause a novel form of juvenile primary aldosteronism. Molecular and Cellular Endocrinology 441 134-139. (https://doi.org/10.1016/j. mce.2016.07.031)

Tischler G \& Leonard S 2014 biobambam: tools for read pair collation based algorithms on BAM files. Source Code for Biology and Medicine $\mathbf{9}$ 13. (https://doi.org/10.1186/1751-0473-9-13)

Tissier F, Cavard C, Groussin L, Perlemoine K, Fumey G, Hagnere AM, Rene-Corail F, Jullian E, Gicquel C, Bertagna X, et al. 2005 Mutations of beta-catenin in adrenocortical tumors: activation of the Wnt signaling pathway is a frequent event in both benign and malignant adrenocortical tumors. Cancer Research 65 7622-7627. (https://doi. org/10.1158/0008-5472.CAN-05-0593)

Vouillarmet J, Fernandes-Rosa F, Graeppi-Dulac J, Lantelme P, DecaussinPetrucci M, Thivolet C, Peix JL, Boulkroun S, Clauser E \&
Zennaro MC 2016 Aldosterone-producing adenoma With a somatic KCNJ5 mutation revealing APC-dependent familial adenomatous polyposis. Journal of Clinical Endocrinology and Metabolism 101 3874-3878. (https://doi.org/10.1210/jc.2016-1874)

Wang K, Li M \& Hakonarson H 2010 ANNOVAR: functional annotation of genetic variants from high-throughput sequencing data. Nucleic Acids Research 38 e164. (https://doi.org/10.1093/nar/gkq603)

Wilm A, Aw PP, Bertrand D, Yeo GH, Ong SH, Wong CH, Khor CC, Petric R, Hibberd ML \& Nagarajan N 2012 LoFreq: a sequencequality aware, ultra-sensitive variant caller for uncovering cellpopulation heterogeneity from high-throughput sequencing datasets. Nucleic Acids Research 40 11189-11201. (https://doi.org/10.1093/nar/ gks918)

Wu VC, Wang SM, Chueh SJ, Yang SY, Huang KH, Lin YH, Wang JJ, Connolly R, Hu YH, Gomez-Sanchez CE, et al. 2017 The prevalence of CTNNB1 mutations in primary aldosteronism and consequences for clinical outcomes. Scientific Reports 7 39121. (https://doi. $\operatorname{org} / 10.1038 /$ srep39121)

Young WF 2007 Primary aldosteronism: renaissance of a syndrome. Clinical Endocrinology 66 607-618. (https://doi. org/10.1111/j.1365-2265.2007.02775.x)

Zennaro MC, Boulkroun S \& Fernandes-Rosa F 2017 Genetic causes of functional adrenocortical adenomas. Endocrine Reviews 38 516-537. (https://doi.org/10.1210/er.2017-00189)

Zheng S, Cherniack AD, Dewal N, Moffitt RA, Danilova L, Murray BA, Lerario AM, Else T, Knijnenburg TA, Ciriello G, et al. 2016 Comprehensive pan-genomic characterization of adrenocortical carcinoma. Cancer Cell 29 723-736. (https://doi.org/10.1016/j. ccell.2016.04.002)

Received in final form 7 February 2019

Accepted 11 February 2019

Accepted Preprint published online 11 February 2019 (c) 2019 Society for Endocrinology Published by Bioscientifica Ltd. Printed in Great Britain 